\title{
PERCEPCIONES SOBRE LA RESPONSABILIDAD SOCIAL CORPORATIVA DE LAS EMPRESAS: RELACIÓN CON LAS ACTITUDES Y CONDUCTAS DE SUS CLIENTES
}

\author{
PERCEPTIONS OF CORPORATE SOCIAL \\ RESPONSIBILITY: RELATIONSHIP WITH \\ CUSTOMERS' ATTITUDES AND BEHAVIORS
}

SERGIO A. PÉREZ-MARTÍNEZ ${ }^{1}$ Y GABRIELA TOPA ${ }^{1}$

Cómo referenciar este artículo/How to reference this article:

Pérez-Martínez, S. A. y Topa, G. (2018). Percepciones sobre la responsabilidad social corporativa de las empresas: relación con las actitudes y conductas de sus clientes [Perceptions of Corporate Social Responsibility: Relationship with Customers' Attitudes and Behaviors]. Acción Psicológica, 15(1), 103-120. https://doi.org/10.5944/ap.15.1.22003

\section{Resumen}

El presente estudio explora la relación entre las percepciones de una muestra de clientes sobre la Responsabilidad Social Corporativa (RSC) de las empresas del IBEX 35 y sus actitudes y conductas. Las percepciones de los clientes sobre la RSC de las empresas del IBEX 35 se relacionan con el beneficio percibido, con el valor percibido de los servicios o productos de la empresa y con la intención de compra o adquisición futura del producto o servicio. La muestra ha estado formada por 1011 clientes de las empresas cotizantes en el IBEX 35. A partir de los resultados se afirma que las tres dimensiones de la RSC percibida por

Correspondencia: Gabriela Topa. Departamento de Psicología Social y de las Organizaciones. Universidad Nacional de Educación a Distancia (UNED).

Email: gtopa@psi.uned.es

ORCID: Sergio Antonio Pérez Martínez (http://orcid.org/0000-0001-5123-3005) y Gabriela Topa (http://orcid.org/0000$\underline{0002-9181-8603)}$

${ }^{1}$ Universidad Nacional de Educación a Distancia (UNED), España.

Recibido: 03 marzo de 2018.

Aceptado: 22 de mayo de 2018. 
los clientes son diferentes entre sí, y que se relacionan positiva y significativamente con el beneficio, el valor percibido y la intención de compra de los clientes. Las aportaciones de este estudio permitirán enlazar las actividades sociales de las empresas y el grado de motivación de los consumidores, relaciones éstas que se convierten en esenciales a la hora del diseño de los productos y / o servicios de las empresas.

Palabras clave: Responsabilidad Social Corporativa; Actitudes; Conducta; Valor percibido; Intención de compra.

\begin{abstract}
The present study aims to empirically explore the relationship between perceptions of Corporate Social Responsibility (CSR) of IBEX 35 companies' customers, their attitudes and behaviors. The relationship between the customers' perception of CSR of the IBEX 35 companies with their products or services' perceived benefit and perceived value and with customers' buying intention of their products or services is empirically analyzed. The sample includes 1011 clients of the IBEX 35 companies. The results supported that the three dimensions of the CSR perceived by the clients are different from each other, and that they are related positively and significantly with the benefit, the perceived value and the customers' purchase intention. The study contributions will link the Firm's CSR activities with the degree of motivation of consumers, relationships that become essential when designing companies' products and / or services.
\end{abstract}

Keywords: Corporate Social Responsibility; Attitudes; Behavior; Perceived value; Buying intention.

\section{Introducción}

La Responsabilidad Social Corporativa (en adelante, RSC) es una cuestión que gana cada día más interés entre los directivos de las empresas y, a la vez, despierta interés académico, en especial por su posible impacto sobre las actitudes y conductas de los clientes (Glavas, 2016a).
Kotler y Lee (2005) señalaban que la satisfacción de las necesidades de los clientes es un proceso social a través del cual grupos e individuos obtienen los bienes y servicios que satisfacen sus necesidades y deseos a la vez que generan, ofrecen e intercambian productos de valor con otras personas.

Los estudios empíricos sobre la relación entre la RSC y las actitudes y conductas de los clientes son variados y la mayoría subraya una estrecha relación entre ambas (Bridoux, Stofberg y Den Hartog, 2016; Frederick, 2016; Glavas, 2016a; Glavas y Kelley, 2014). Desde hace ya un par de décadas se ha puesto de manifiesto que una percepción negativa de la RSC de una empresa podía tener impacto duradero en la lealtad de sus usuarios y en sus decisiones de compra o uso de los servicios que esta presta. Como ejemplo, se podría citar la publicidad relativa al empleo de mano de obra infantil o semi esclavizada por parte de empresas como Nike o Adidas y la consiguiente movilización de sus clientes para boicotearles.

Para apoyar el extremo positivo de esta relación, es decir la influencia favorable de una percepción sobre la RSC en la lealtad y conducta de compra del cliente, se acumulan evidencias a través de un creciente número de trabajos empíricos (Hameed, Riaz, Arain y Farooq, 2016; Jones, Willness y Glavas, 2017; Voliotis, Vlachos y Epitropaki, 2016; Wang, Fu, Qiu, Moore y Wang, 2017). Este interés renovado se observa por el incremento reciente de investigaciones en la materia, muchas de ellas agrupadas en el monográfico coordinado por Glavas, Willness y Jones (2017).

Son diversos los enfoques teóricos que explican esta relación. Uno de los más relevantes se centra en cómo la imagen de la RSC de la empresa es usada por los clientes y consumidores a la hora de tomar decisiones sobre la compra y el consumo de bienes y servicios (Glavas, 2016b). Grandes empresas internacionales hacen esfuerzos por construir y mantener una imagen socialmente responsable a partir de tres dimensiones. En primer lugar, su asociación estratégica con causas sociales. En segundo, sus opciones favorables a un desarrollo sostenible. Por último, sus decisiones éticamente responsables a la hora de garantizar la continuidad de sus beneficios comerciales. 
La investigación empírica en España sobre la relación entre la percepción de la RSC de la empresa y las actitudes $\mathrm{y}$ conductas de los clientes es escasa (Moreno, Uriarte y Topa, 2010) y se ha centrado mayoritariamente en sectores específicamente destinados a la prestación de servicios (Fisac, Moreno, Mataix y Palacios 2011). En relación con las empresas del IBEX 35, que poseen una elevada visibilidad en sus respectivos sectores, se ha hecho importante investigación, pero esta se ha enfocado desde perspectivas económicas, o bien se han subrayado el papel de las presiones externas que motivan las prácticas de RSC (PérezLópez, Moreno-Romero y Barkemeyer, 2015).

En este sentido, parece importante analizar las percepciones de los clientes en relación con las diversas dimensiones de la RSC, a fin de valorar su posible impacto en las actitudes y conductas futuras de dichos clientes. Así, el objetivo del presente estudio es explorar empíricamente la relación entre las percepciones de RSC de una muestra de clientes de las empresas del IBEX 35 y el valor percibido de los servicios o productos, el beneficio percibido con su adquisición y la intención de compra o adquisición futura del producto o servicio. Las aportaciones de este estudio permitirán enlazar las actividades sociales de las empresas y el grado de motivación de los consumidores, relaciones estas que se convierten en esenciales a la hora del diseño de los productos y/o servicios de las empresas (Barone, Miyazaki y Taylor, 2007; Simmons y Becker-Olsen, 2006).

\section{Percepción de la RSC: tres dimensiones del desarrollo sostenible}

Los primeros trabajos empíricos, que aparecen en los años 90 , de una forma mayoritaria sugieren una positiva relación entre las acciones de RSC y las actitudes del consumidor (Brown y Dacin, 1997; Creyer y Ross, 1997; Becker-Olsen, Cudmore y Hill, 2006). Al principio, sin embargo, el concepto de RSC se limitaba a los aspectos económicos, con base legal y de una forma filantrópica. $\mathrm{La}$ RSC moderna tiene muchas definiciones, que incluyen implícita o explícitamente un fin económico. Pava y Krausz (1995) definen a una organización que asuma sus responsabilidades para la sociedad más allá de la maximización de sus propias ganancias. Otra definición más específica de la RSC indica que la forma de operar en un negocio ha de efectuarse de una manera que cumpla o exceda las expectativas éticas, legales, comerciales y públicas que la sociedad tiene de los negocios (Kotler y Lee, 2005). En el marco del presente estudio, la definición de la RSC se centra en el compromiso de la empresa para maximizar el beneficio económico a largo plazo, el bienestar social y el desarrollo medioambiental sostenible a través de sus prácticas, políticas y recursos comerciales (Alvarado, Bigne, Aldas y Currás, 2016; Du, Bhattacharya y Sen, 2010)

Son otros autores posteriores los que avanzarán en el tema, poniendo énfasis en cuándo, cómo y para qué acciones sociales específicas producen asociaciones positivas entre RSC y el comportamiento del consumidor (Sen y Bhattacharya, 2001). Entre los estudios empíricos que vinculan la RSC y las actitudes y conductas de los clientes cabe destacar el trabajo de Pomering y Dolnicar (2009), basado en sus encuestas de mercado. En éste pusieron de manifiesto que los consumidores atienden a la información brindada por las organizaciones y que una gran mayoría apoyaría las iniciativas de RSC de dichas compañías. En el mismo sentido, los trabajos de Mohr y Webb (2005) demostraron que la RSC tiene un considerable impacto en las respuestas de los consumidores. Del mismo modo, otros autores indican que una comunicación constante, así como una difusión de información sobre actividades de RSC son clave para mantener a la sociedad consciente de los comportamientos de la empresa al respecto (McWilliams y Siegel, 2001). Así, se podría decir que el propósito principal de la RSC es contribuir al éxito de las empresas que participan en tales iniciativas de RSC (Batruch, 2010). De acuerdo con Kusum y Luan (2011) a la pregunta de si "¿La Responsabilidad Social Corporativa genera lealtad con los clientes?", la respuesta parece ser afirmativa. Por el contrario, las percepciones negativas de los usuarios sobre la RSC de las empresas parece que obstaculizan el patrocinio de los clientes (Dodd y Supa, 2011).

En definitiva, hay un creciente número de investigaciones empíricas que indican una asociación positiva entre una política de RSC, y las actitudes y conductas de sus clientes (Hameed et al., 2016; Jones et al., 2017; Voliotis et al., 2016; Wang et al., 2017). Sus resultados sugieren que los consumidores esperan que las empresas participen en iniciativas sociales y que, por ello, las recompensan por 
sus esfuerzos a través del comportamiento de compra. Finalmente, estos estudios constatan el efecto positivo de las acciones de RSC sobre el valor de marca cuando dichas acciones son percibidas como altruistas y no como egoístas por parte del consumidor.

\section{Valor percibido y Beneficio percibido}

Aunque se han llevado a cabo muchos estudios con conclusiones significativas sobre la RSC centrada en estrategia de negocios, en el análisis de partes interesadas y en la ventaja competitiva, con respecto al beneficio y al valor percibido la literatura aborda este sector con poca frecuencia. Considerando el valor percibido se puede distinguir a una empresa de su competencia en el mercado (Quintão e Isabella, 2015). El valor percibido por el consumidor delante de una oferta de producto o servicio es el resultado de una comparación de los beneficios que recibe en la transacción que realiza con la empresa y los sacrificios o costes (financieros o no) que debe invertir en dicho intercambio (Quintão e Isabella, 2015). Como el valor percibido de una compra es un antecedente de la intención de compra, no es difícil de entender que, si la RSC influye en el valor percibido, pueda hacerlo también en la intención de compra. El beneficio percibido representa las ganancias que el consumidor obtiene por su intercambio con la empresa. Aunque muchas definiciones del valor y del beneficio percibido han usado sólo la calidad como indicador de beneficio, los usuarios y consumidores pueden considerar también otros aspectos relativamente importantes para ellos como los beneficios emocionales (Ferreira, Avila y Dias, 2010).

\section{Intención de compra o uso futuro del bien o servicio}

La intención de compra puede entenderse más fácilmente como la estimación del cliente acerca de la probabilidad de que adquiera el servicio o producto en el futuro (Quintão e Isabella, 2015). Debido a que la mayoría de los estudios en RSC se centran en su incidencia sobre los resultados económicos de las empresas, la literatura no refleja tan claramente las intenciones de compra en relación con la RSC. En este sentido, cabe destacar a este respecto el estudio de Gold, Seuring y Beske (2010), donde se muestra cómo la información positiva y negativa sobre la responsabilidad social y ambiental de las empresas influye en las intenciones de compra, empleo e inversión de las diversas partes interesadas. En el diseño de este estudio se aplicaron dos condiciones. En el tratamiento de una "RSC positiva", el desempeño social y ambiental de la compañía se describió con un fuerte desempeño social, mientras que en el tratamiento de una "RSC negativa" se describió con una perspectiva de desempeño social débil. Asimismo, las intenciones de los encuestados de comprar productos, de búsqueda de empleo y de invertir en la empresa se evaluaron mediante escalas autoinformadas. Los resultados muestran que la información positiva de la RSC sobre una empresa mejora las intenciones de los consumidores de comprar productos, también las intenciones de los potenciales empleados de búsqueda de empleo y la intención de las potenciales inversiones en la compañía. Para sintetizar, aunque la evidencia empírica es todavía limitada, la relación entre la RSC percibida por los clientes y sus intenciones de compra parece consistente.

En resumen, sobre la base de la literatura revisada hasta aquí, en el presente estudio se proponen las hipótesis, que se grafican también en la Figura 1.

Hipótesis 1: las percepciones de los clientes sobre la RSC de las empresas, en sus tres dimensiones de Protección medioambiental (H1a), Equidad social (H1b), y desarrollo económico sostenible (H1c), se relacionarán positivamente con el valor percibido de productos o servicios de la empresa.

Hipótesis 2: las percepciones de los clientes sobre la RSC de las empresas, en sus tres dimensiones de Protección medioambiental (H2a), Equidad social (H2b), y desarrollo económico sostenible $(\mathrm{H} 2 \mathrm{c})$, se relacionarán positivamente con el beneficio percibido de productos o servicios de la empresa.

Hipótesis 3: las percepciones de los clientes sobre la RSC de las empresas, en sus tres dimensiones de Protección medioambiental (H3a), Equidad social (H3b), y desarrollo económico sostenible ( $\mathrm{H} 3 \mathrm{c})$, se relacionarán positivamente con la intención de compra de productos o servicios de la empresa. 


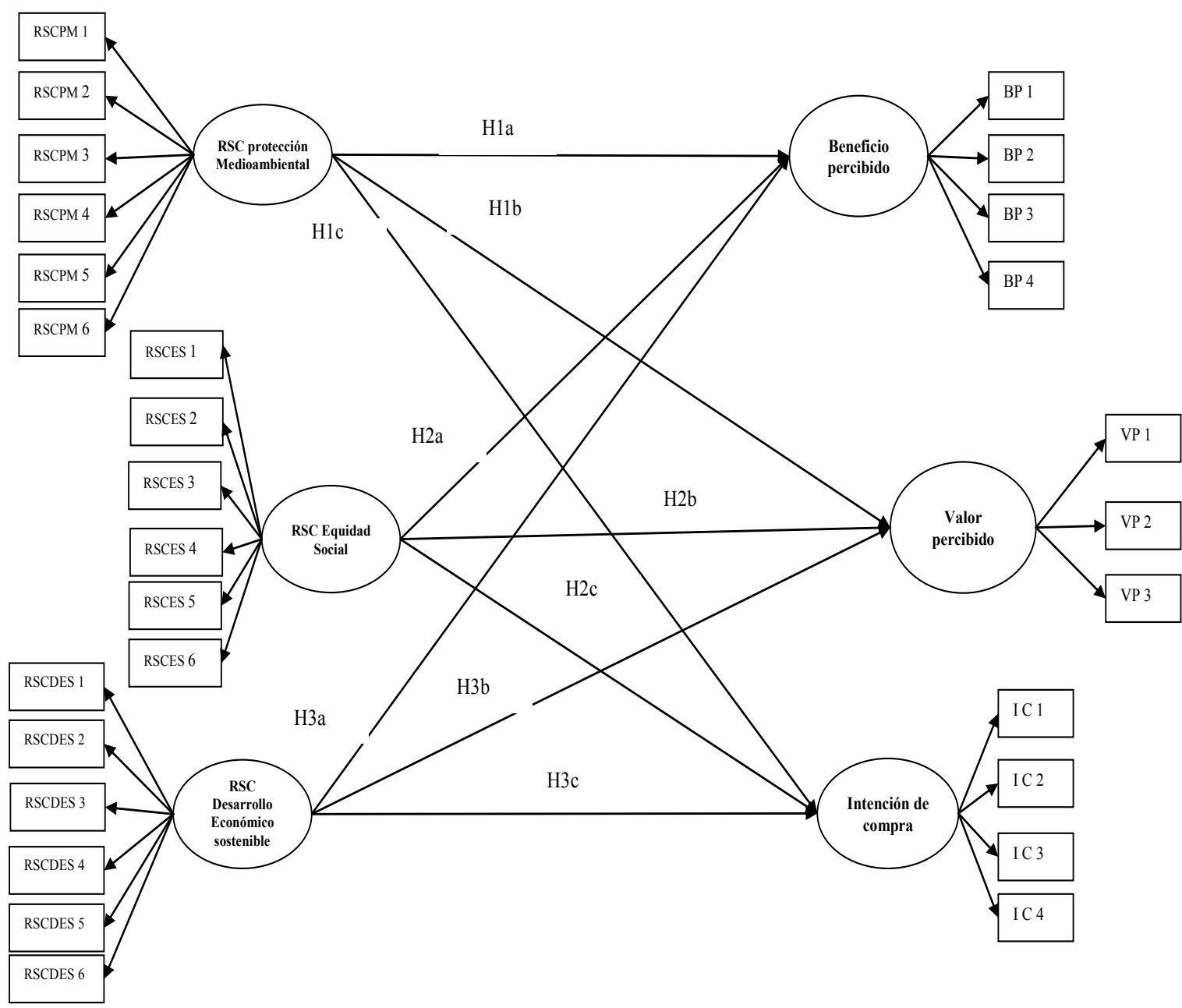

Figura 1. Modelo hipotetizado

\section{Método}

\section{Participantes}

El presente estudio se ha llevado a cabo con la participación de 1011 clientes de las empresas cotizantes en el IBEX 35. La media de edad de los participantes fue 40.4 años (D. T.: 12.6), el $48 \%$ son varones, la antigüedad media como clientes de la empresa fue de 14.3 años (D. T.: 32.3). Por lo que se refiere a la empresa acerca de la cual respondían, $28.8 \%$ se referían a diferentes bancos, el $18.7 \%$ lo hicieron sobre un grupo textil, $12 \%$ acerca de cadenas de supermercados, $15.3 \%$ acerca de empresas de telefonía, 9.2\% acerca de empresas del sector de la energía, y $1.5 \%$ acerca de empresas de construcción y obra pública. En relación con su nivel de estudios, $12.9 \%$ tenían estudios básicos, $21 \%$ habían finalizado el bachillerato, $39 \%$ alcanzaron estudios medios, y $26.9 \%$ eran licenciados o ingenieros. El $64 \%$ trabajaba a tiempo completo, el $16 \%$ trabajaba a tiempo parcial y el $19.7 \%$ estaba 
desempleado. En cuanto al sector en que trabajaban, $39.1 \%$ lo hacían en empresas de servicios, $11.8 \%$ en la industria, $9.1 \%$ en la sanidad, $8.6 \%$ en la educación, $10.9 \%$ en la banca y las telecomunicaciones. En relación con la categoría profesional de sus puestos de trabajo, un $5 \%$ eran directivos, $10.1 \%$ eran mandos intermedios, $66.9 \%$ eran empleados cualificados y $17.9 \%$ eran empleados no cualificados.

\section{Procedimiento}

Los participantes fueron reclutados entre la población general de personas mayores de 18 años, a quienes se les envió un email de invitación a participar anónima y voluntariamente, con el vínculo a la encuesta online. Las invitaciones fueron enviadas por los colaboradores del equipo investigador a sus contactos personales, sin que los investigadores principales tuvieran acceso a las direcciones de email de los potenciales participantes, a fin de mantener el anonimato del estudio. El único criterio de inclusión fue la consideración de sí mismos como clientes de alguna de las empresas cotizantes en el IBEX 35, cuyo listado se adjuntaba al email. Los colaboradores informaron a los participantes de los objetivos del estudio, del anonimato de su participación, de la voluntariedad y gratuidad de su participación, de la confidencialidad de las informaciones que brindasen, y de la posibilidad de abandonarlo en cualquier momento sin repercusiones negativas. Del total de invitaciones enviadas $(\mathrm{N}=1407), \quad$ se obtuvieron 1139 respuestas (tasa de respuesta del $81 \%$ ), pero se eliminaron 128 encuestas a partir del análisis de las dos preguntas de control (Careless response items) introducidas en el cuestionario. De acuerdo con los procedimientos habituales, se solicitó la pertinente autorización al Comité de Bioética de la UNED para el presente estudio y éste fue aprobado.

\section{Instrumentos de evaluación}

\section{$R S C$ percibida por los clientes}

La escala Corporate Social Responsibility Perception Scale (CSRPerScale) se utilizó para evaluar las percepcio- nes de los clientes acerca de la actuación socialmente responsable de las empresas en tres dimensiones de Protección medioambiental, Equidad social y Desarrollo económico sostenible. La escala original, desarrollada por Alvarado, Bigne, Aldas y Curras (2017), contiene seis ítems para cada dimensión. Ejemplos de ítems son los siguientes: En mi opinión, en relación con el medioambiente, la empresa ... está tratando de llevar adelante programas para reducir la contaminación, En mi opinión, en relación con el medioambiente, la empresa ...está tratando de llevar adelante programas para usar exclusivamente los recursos naturales imprescindibles (Protección medioambiental), En mi opinión, en relación con la sociedad, la empresa ...realmente está tratando de apoyar programas culturales, En mi opinión, en relación con la sociedad, la empresa ...realmente está totalmente comprometida con unos principios éticos bien definidos (Equidad social), En mi opinión, en relación con la economía, la empresa ...realmente está tratando de construir sólidas relaciones con sus clientes para asegurar su éxito a largo plazo, En mi opinión, en relación con la economía, la empresa ...realmente está tratando de mejorar continuamente la calidad de los servicios que ofrece (Desarrollo económico sostenible).

En el estudio original de sus desarrolladores, la fiabilidad de las dimensiones fue adecuada, ya que en todos los casos superó el .90 (Protección medioambiental $\alpha=.95$, Equidad social $\alpha=.92$, y Desarrollo económico sostenible $\alpha=.96$ ). La escala de respuesta original, tipo Likert sin punto medio, se adaptó en el presente estudio para incluir el valor medio de la de indiferencia, oscilando las posibles respuestas entre 1 (Totalmente en desacuerdo) y 5 (Totalmente de acuerdo).

\section{Beneficio percibido}

Para evaluar esta variable se usó la escala de Ferreira, Avila y Faria (2010), con cuatro ítems, referidos a los beneficios que les producía la adquisición de productos o servicios de la empresa acerca de la cual respondían. La escala de respuesta fue tipo Likert, con cinco opciones oscilando las posibles respuestas entre 1 (Totalmente en desacuerdo) y 5 (Totalmente de acuerdo), e incluía el punto medio neutral. Ejemplos de los ítems son: Comprar un producto de la empresa...me hace sentir bien conmigo 
mismo, Comprar un producto de la empresa...me hace sentir que estoy beneficiándome. En el estudio de Quintâo e Isabella (2015), la escala mostró una adecuada fiabilidad $(\alpha=.92)$.

\section{Valor percibido}

Para evaluar esta variable se usó la escala de Ferreira, Avila y Faria (2010), con tres ítems, referidos al valor de los productos o servicios de la empresa acerca de la cual respondían. La escala de respuesta fue tipo Likert, con cinco opciones oscilando las posibles respuestas entre 1 (Totalmente en desacuerdo) y 5 (Totalmente de acuerdo), e incluía el punto medio neutral. Ejemplos de los ítems son: Si comprase un producto de la empresa...estaría recibiendo lo que mi dinero vale, Comprar un producto de la empresa...es una compra que vale la pena por la relación calidad precio. En el estudio de Quintâo e Isabella (2015), la escala mostró una adecuada fiabilidad $(\alpha=.88)$, pese a su reducida longitud.

\section{Intención de compra}

Para evaluar esta variable se usó la escala de Ferreira, Avila y Faria (2010), con cuatro ítems, referidos a la intención de adquisición en el futuro de productos o servicios de la empresa acerca de la cual respondían. La escala de respuesta fue tipo Likert, con cinco opciones oscilando las posibles respuestas entre 1 (Totalmente en desacuerdo) y 5 (Totalmente de acuerdo), e incluía el punto medio neutral. Ejemplos de los ítems son: Estoy dispuesto a comprar un producto de la empresa..., La probabilidad de comprar un producto de la empresa en el futuro...es alta. En el estudio de Quintâo e Isabella (2015), la escala mostró una adecuada fiabilidad $(\alpha=.92)$.

Datos sociodemográficos: diversas preguntas relativas a las características sociodemográficas de los participantes, tales como el género, la edad, los años de antigüedad como cliente de la empresa acerca de la cual responden los participantes.

Preguntas de control (Careless response items). Una de las desventajas potenciales de las encuestas online es que los participantes no pueden ser vigilados adecuadamente y pueden responder a las preguntas de forma poco atenta. Como forma de vigilancia de las respuestas poco atentas, o descuidadas, Meade y Craig (2012) recomiendan adoptar diversos procedimientos. Uno de ellos consiste en incluir algunos ítems falsos a fin de asegurarse que los participantes responden prestando atención a aquello que se les pregunta. Estos ítems falsos tienen una sola respuesta correcta y la elección de las respuestas incorrectas sugiere que el participante no está tomando el estudio en serio. En el presente estudio se usaron dos tipos de ítems: Estoy leyendo con cuidado las preguntas de esta encuesta, Estoy pasando a toda velocidad este cuestionario. Estos ítems se mezclaron en medio de la escala de RSC percibida por los clientes debido su importancia para esta investigación. Después de filtrar las respuestas para detectar a los participantes con un estilo de respuesta desatenta, aquellos que puntuaron 3 o menos en la primera pregunta, y 3 o más en la segunda, fueron eliminados.

\section{Análisis de datos}

El análisis de datos se llevó a cabo con el programa Smart PLS v. 3.2.6. (Ringle, Wende, y Becker, 2015), que estima los coeficientes de regresión estandarizados para las diferentes relaciones establecidas en el modelo entre los indicadores observables y las variables latentes, así como entre las diferentes variables latentes. De esta forma se obtienen dos tipos de resultados. En primer lugar, se obtienen los indicadores acerca de las propiedades psicométricas de los constructos presentes en las hipótesis del estudio (modelo de medida). En segundo lugar, se pueden analizar simultáneamente relaciones de predicción entre distintas variables latentes (modelo estructural). La opción por un modelo de ecuaciones estructurales, y en concreto por la técnica de mínimos cuadrados parciales (Partial Least Squares, PLS) se debió a que las variables no necesariamente cumplen los supuestos exigidos en otros métodos. Para valorar la significación de las relaciones en el modelo estructural se recurrió al bootstrapping de 500 muestras, lo que lleva a un valor crítico de $t$ de 1.96 para una $p<.05$. 
Tabla 1

Estadísticos descriptivos y correlaciones

\begin{tabular}{|c|c|c|c|c|c|c|c|c|}
\hline Variables & $M$ & $D T$ & 1 & 2 & 3 & 4 & 5 & 6 \\
\hline 1.RSC Protección Medioambiental & 2.79 & .75 & .72 & & & & & \\
\hline 2. RSC Equidad Social & 2.77 & .80 & $.71^{* *}$ & .80 & & & & \\
\hline 3. RSC Desarrollo Económico sostenible & 2.84 & .78 & $.70^{* *}$ & $.67^{* *}$ & .76 & & & \\
\hline 4. Beneficio percibido & 3.13 & .84 & $.44^{* *}$ & $.35^{\star *}$ & $.48^{* *}$ & .86 & & \\
\hline 5. Valor percibido & 3.00 & .98 & $.41^{* *}$ & $.32^{* *}$ & $.51^{* *}$ & $.65^{\star *}$ & .92 & \\
\hline 6. Intención de compra & 3.46 & .97 & $.36^{* *}$ & $.30^{* *}$ & $.51^{* *}$ & $.59^{* *}$ & $.66^{\star *}$ & .91 \\
\hline
\end{tabular}

Nota. $\mathrm{N}=1011,{ }^{* *} p<.01$. Los valores de la diagonal muestran la raíz cuadrada de AVE.

\section{Resultados}

En primer lugar, se calcularon las correlaciones entre las variables de estudio (ver Tabla 1).

\section{Análisis de la Modelización con PLS}

Este análisis se realizó en dos etapas: en primer lugar, se analizó la fiabilidad, la validez convergente y discriminante del modelo de medida y a continuación se valoró el modelo estructural, es decir, en qué medida la percepción por parte de los clientes de la conducta socialmente responsable de las empresas permitía predecir el beneficio Tabla 2

Modelo de medida: fiabilidad y validez convergente percibido, el valor percibido de los productos o servicios y la intención de compra futura de los clientes.

Modelo de medida. Se comprobó la fiabilidad individual a través de la significación estadística de las cargas factoriales estandarizadas $(\lambda)$ de los indicadores de cada constructo latente. Todos los ítems superaban los niveles recomendados de .60 (Hair, Black, Babin, Anderson y Tatham, 2006), como se aprecia en la Tabla 2.

Para evaluar la fiabilidad de las medidas se calculó tanto el coeficiente de fiabilidad compuesta (CFC) como el alfa de Cronbach. Mientras que el alfa de Cronbach presupone que cada indicador de un constructo contribuye de

\begin{tabular}{|c|c|c|c|c|c|c|c|c|c|c|c|}
\hline Variable latente & Ítem & $\lambda$ & CFC & $\alpha$ & AVE & Variable latente & Ítem & $\lambda$ & CFC & $\alpha$ & AVE \\
\hline RSC Protección & E1. & .77 & \multirow[t]{6}{*}{.87} & \multirow{6}{*}{.82} & \multirow{6}{*}{.52} & \multirow{4}{*}{$\begin{array}{l}\text { Beneficio } \\
\text { percibido }\end{array}$} & B01. & .89 & \multirow[t]{4}{*}{.92} & \multirow[t]{4}{*}{.88} & \multirow[t]{4}{*}{.74} \\
\hline \multirow[t]{5}{*}{ Medioambiental } & E2. & .76 & & & & & B02. & .84 & & & \\
\hline & $\mathrm{E} 3$ & 75 & & & & & B03. & 88 & & & \\
\hline & E4. & .70 & & & & & B04. & .83 & & & \\
\hline & E5. & 69 & & & & \multirow{2}{*}{ Valor percibido } & VP1. & .92 & \multirow{2}{*}{.94} & \multirow{2}{*}{.90} & \multirow{2}{*}{.83} \\
\hline & E6. & .65 & & & & & VP2. & .93 & & & \\
\hline \multirow{6}{*}{$\begin{array}{l}\text { RSC Equidad } \\
\text { Social }\end{array}$} & S1. & .84 & \multirow[t]{6}{*}{.91} & \multirow[t]{6}{*}{.88} & \multirow[t]{6}{*}{.64} & \multirow{5}{*}{$\begin{array}{l}\text { Intención de } \\
\text { compra }\end{array}$} & VP3. & .90 & \multirow{5}{*}{.95} & \multirow{5}{*}{.93} & \multirow{5}{*}{.83} \\
\hline & S2. & .82 & & & & & IC1. & .92 & & & \\
\hline & S3. & .80 & & & & & IC2. & .94 & & & \\
\hline & S4. & .81 & & & & & IC3. & .92 & & & \\
\hline & S5. & .76 & & & & & IC4. & .86 & & & \\
\hline & s6. & .77 & & & & & & & & & \\
\hline RSC Desarrollo & EC1. & & .87 & .85 & .58 & & & & & & \\
\hline Económico & EC2. & .86 & & & & & & & & & \\
\hline sostenible & EC3 & .80 & & & & & & & & & \\
\hline & EC4. & $\begin{array}{r}.74 \\
65\end{array}$ & & & & & & & & & \\
\hline & EC6. & .63 & & & & & & & & & \\
\hline
\end{tabular}




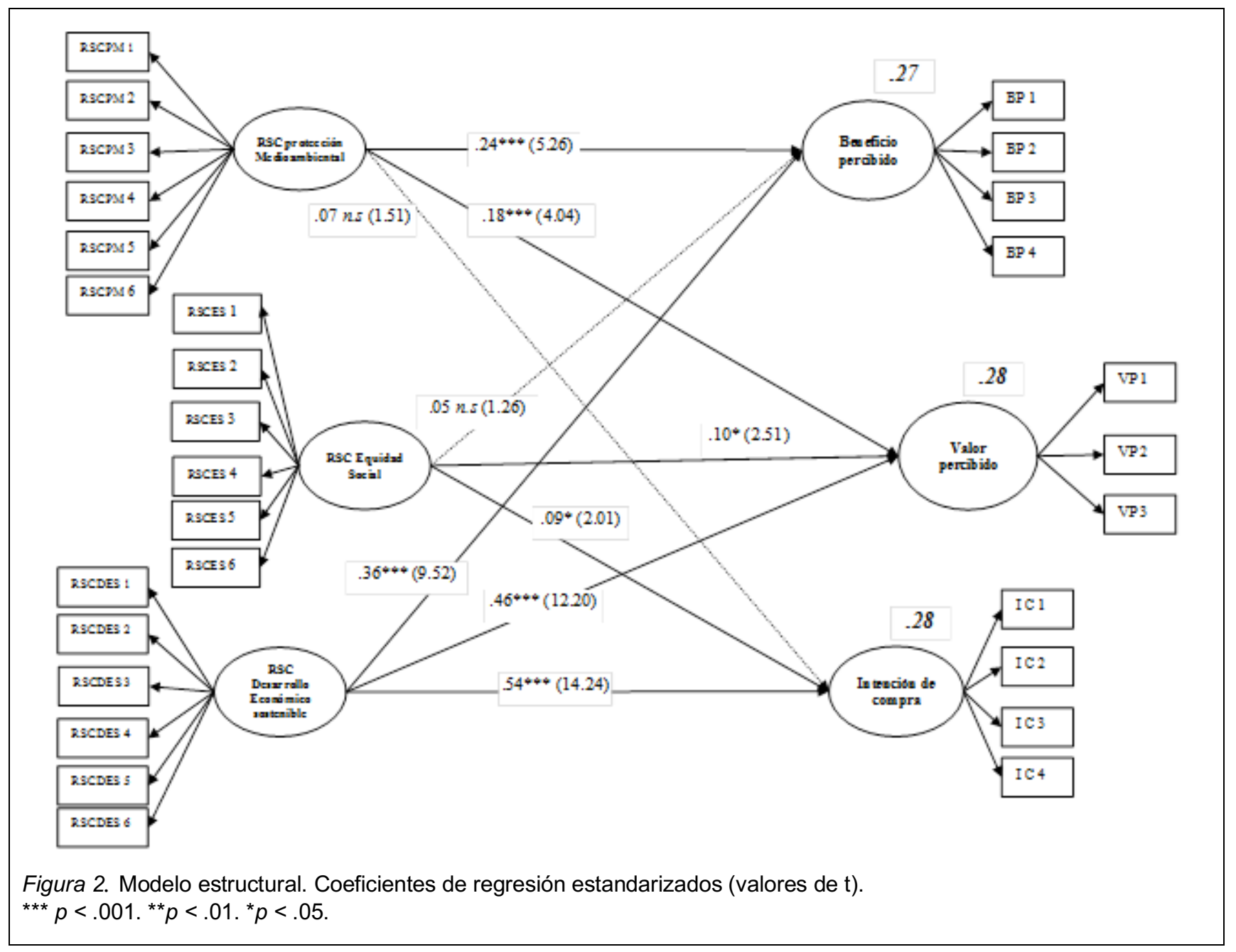

la misma forma, la fiabilidad compuesta utiliza las cargas de los ítems del modelo causal. Todos los valores se consideran adecuados ya que superan el .70.

La validez convergente se midió a través de la varianza media extraída (Average Variance Extracted, AVE) que refleja la cantidad total de la varianza de los indicadores recogida por la variable latente. Cuanto mayor sea el valor de AVE, más representativos son los indicadores de la variable latente correspondiente. El valor mínimo recomendado es .50, es decir, que más del $50 \%$ de la varianza del constructo sea explicada por sus indicadores. En este estudio, todas las variables latentes superaron los valores aceptados de AVE (véase Tabla 2).
Para determinar la validez discriminante entre constructos, es necesario que la raíz cuadrada del AVE sea superior a la correlación entre constructos. En la Tabla 1 se presentan las correlaciones entre constructos y, en la diagonal, la raíz cuadrada del AVE. Teniendo en cuenta la tabla anterior, se puede afirmar que existe validez discriminante entre todos los constructos del modelo teórico propuesto. El criterio de validez discriminante requiere que la raíz cuadrada del AVE de cada constructor sea superior a la correlación de ese constructo con los otros constructos y que las correlaciones entre los distintos factores no superen 80 , lo que ocurre en este caso para todas las variables del modelo, aunque la correlación entre las tres 
subdimensiones de la percepción de la RSC son altas, como es de esperar.

Modelo estructural. En este modelo se incluyeron las relaciones entre la percepción de RSC de Protección medioambiental, la percepción de RSC de Equidad Social, la percepción de RSC de Desarrollo económico - de una parte- $y$ el beneficio percibido, el valor percibido y la intención de compra de los clientes, de la otra parte. El modelo permitiría explicar un $26.6 \%$ de la varianza del beneficio percibido, un $28.1 \%$ del valor percibido y un $28.4 \%$ de la intención de compra de los clientes. Sin embargo, pese a que la mayoría de las relaciones propuestas entre las dimensiones de la RSC y los resultados alcanzaron la significación estadística, dos de ellas no fueron significativas.

Como se refleja en la Figura 2, las relaciones entre la RSC de Protección medioambiental y la intención de compra no son significativas. Lo mismo sucede con las relaciones entre la RSC de Equidad social y el beneficio percibido por los clientes).

\section{Matriz de Importancia y rendimiento de las variables predictoras}

Con el fin de sugerir intervenciones dirigidas a aumentar el beneficio percibido, el valor percibido y la intención de compra se recomienda el Análisis de Matriz de Importancia-Rendimiento (IPMA) de las variables predictoras en los modelos PLS. Para cada variable latente específica, IPMA contrasta la importancia y el rendimiento de sus predictores, lo cual sirve para resaltar las potenciales áreas para la mejora. El objetivo principal es identificar aquellos predictores de una variable con una importancia relativamente alta pero un rendimiento relativamente bajo. Como se muestra en la Figura 3, el IPMA mostró que la RSC de Equidad social es el predictor más importante para entender el beneficio percibido por los clientes. Sin embargo, su rendimiento es claramente inferior al promedio en comparación con los otros constructos, específicamente con la RSC de desarrollo económico sostenible. Por otra parte, en la Figura 3 se observa que, a la hora de pronosticar la intención de compra de los clientes, se repite el escaso
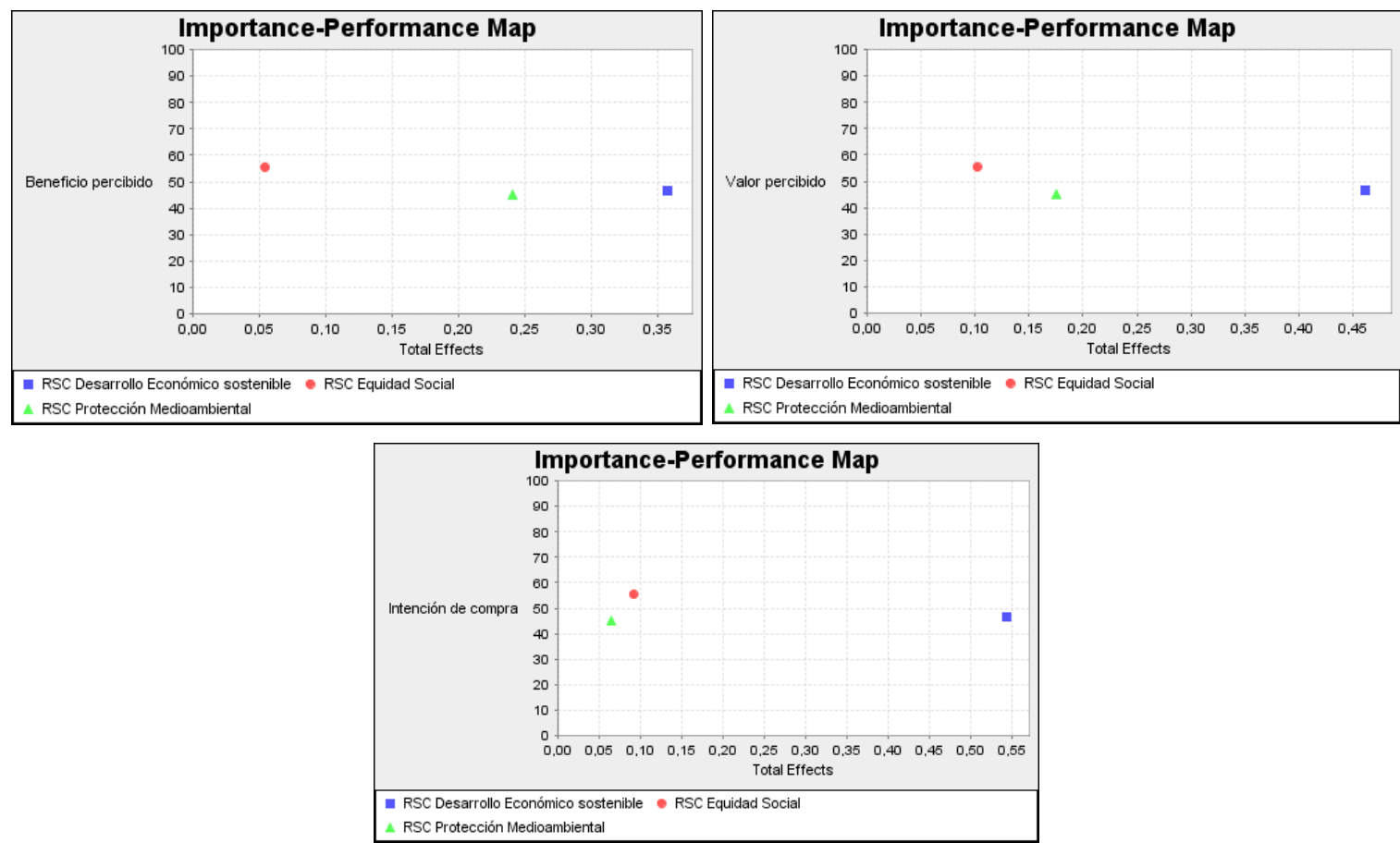

Figura 3. Matriz de importancia y rendimiento de las tres dimensiones de la RSC. 
desempeño de la RSC de Equidad social y protección medioambiental, mientras que la RSC de desarrollo económico se observa un alto rendimiento relativo, aunque esta dimensión tenga menor importancia. En consecuencia, estos resultados podrían proporcionar información adicional sobre cómo incidir en las valoraciones de los clientes y en sus intenciones de compra futura a partir de la información que se brinda sobre las distintas dimensiones de la RSC de las empresas.

\section{Discusión}

El presente estudio tenía por objeto analizar empíricamente, y desde una perspectiva psicosocial, la relación entre la percepción de la RSC en sus tres dimensiones de Protección medioambiental, Equidad social y Desarrollo económico sostenible, por parte de los clientes de las empresas del IBEX 35 con su relación con el beneficio percibido, con el valor percibido de los servicios o productos de la empresa, y con la intención de compra o adquisición futura del producto o servicio por parte de los referidos clientes. A la vista de los resultados obtenidos se puede afirmar que el objetivo se ha alcanzado. El modelo hipotetizado, que se ha se puesto a prueba con 1011 clientes, muestra que las tres dimensiones de la RSC percibida por los clientes son diferentes entre sí, y que se relacionan positiva y significativamente con el beneficio, el valor percibido y la intención de compra, si bien es cierto que algunos resultados discrepantes merecen de una discusión más detallada.

En primer lugar, los hallazgos ponen de manifiesto que la RSC percibida en su dimensión Desarrollo económico sostenible es el mejor predictor de las tres variables de resultado del modelo, y que su influencia es estadísticamente significativa sobre todas ellas, aunque es superior sobre la intención de compra. En el caso de la dimensión de Equidad social, sucede precisamente lo contrario, ya que es un predictor de significación estadística menor en su relación con el valor percibido y la intención de compra, mientras que carece de significación su relación con el beneficio percibido. La percepción de RSC de Protección medioambiental es predictor significativo del beneficio y del valor percibido, pero no predice significativamente la intención de compra. Dado que las dos dimensio- nes de la RSC relativas a la Protección Medioambiental y a la Equidad social tienen relaciones poco significativas con la intención de compra, esta variable resulta predicha en su mayor parte tan sólo por la RSC de Desarrollo económico sostenible.

\section{Implicaciones prácticas}

Aunque el núcleo de la RSC esté relacionado con las responsabilidades de las compañías más allá de la maximización de los beneficios, es cierto que la participación de una organización en prácticas socialmente responsables y sus efectos directos en los comportamientos de sus clientes aún no se han determinado de manera concluyente. Por esta razón intentamos con este estudio que su incidencia quede mejor sustentada. Es importante que reconozcamos, no obstante, la relación entre las intenciones de compra de los consumidores y la participación de las organizaciones en programas socialmente responsables, ya que la RSC a menudo se utiliza a ésta como una simple herramienta de marketing. El cliente es otra pieza vital en la apuesta por un consumo socialmente responsable y sostenible que puede influenciar en la demanda sobre la oferta. La importancia de conocer las expectativas de los consumidores, hacen que, en este contexto, se apueste por intentar aumentar la confianza de los consumidores, una política de RSC activa se reflejará en una influencia positiva en la valoración global y percepción del producto o del servicio ofrecido por las empresas. Por último, la búsqueda de la satisfacción del cliente fomentaría también otras facetas como el impulso de buenas prácticas medioambientales entre todos los agentes de la sociedad, de este modo se iniciarían unas políticas de sostenibilidad, de inversiones socialmente responsables donde primara un sistema ágil de gestión,

Durante mucho tiempo hemos podido comprobar que las organizaciones han tenido un único objetivo principal que no era otro que el de maximizar sus beneficios con una responsabilidad que se fijaba en el ámbito meramente económico. Esta visión ha ido evolucionando hacia un compromiso social. Es esencial que a la hora de involucrar activamente a una empresa con la sociedad se produzca un impulso de la RSC, y que se este pueda ser considerado como un elemento fundamental en la planificación estra- 
tégica de la totalidad de las compañías muchas grandes compañías

Teniendo en cuenta que la RSC tenemos que entenderla como un concepto transversal y donde su aplicación debe consolidarse en el largo plazo, su visión global incumbe a diferentes ámbitos de la gestión de la empresa, que pueden ir por su parte desde las condiciones laborales, la protección de la salud, la lucha contra el fraude y de cómo sentirse involucrado el trabajador o consumidor con la misión y valores de las empresas con las que nos relacionamos, entre otros aspectos. El fomento de una inversión con impacto positivo en la sociedad no hace sino potenciar la reputación y la imagen de la compañía, la consolidación de un escenario sostenible, la creación de valor a largo plazo y dibujar un escenario donde se involucren a los diferentes grupos de interés como accionistas, consumidores, trabajadores, empleadores, inversores...

Es cierto que ha quedado demostrado que muchas empresas han venido impulsando el reparto de parte de sus beneficios en actividades de carácter social, pero el concepto de la RSC debería tener una dimensión más amplia, todas estas acciones con carácter social y medioambiental deben de establecerse de forma voluntaria y de carácter permanente en su aplicación. El establecimiento de estas acciones ha de ir ligado también a la pequeña y mediana empresa, en su aplicación se han de tener en cuenta aspectos como la integración de acciones ligadas a un compromiso real, a un aumento de la transparencia, a una mayor confianza por parte del consumidor, a un respeto por el medio ambiente y a una aplicación de unos criterios éticos que sean compatibles con la innovación y la competitividad empresarial.

La Responsabilidad Social ha de ir más allá de una mera filantropía, su integración en el modelo de negocio de las empresas debería estar fijado como un porcentaje de compromiso con respecto a sus beneficios, de este modo el desarrollo de estas prácticas no sólo no haría un mundo más justo, sino que fomentarían los compromisos de calidad, de honestidad y de transparencia que actualmente adolece nuestra sociedad. Vincular estas actividades a la gestión básica de la empresa y transmitir a la sociedad la misión y valores de una compañía con práctica de RSC es una de las misiones de la sociedad actual para la consoli- dación de aspectos en ámbitos como en la formación, en educación, medioambiente, salud y calidad en el mundo laboral.

\section{Referencias}

Alvarado-Herrera, A., Bigne, E., Aldas-Manzano, J. y Curras-Perez, R. (2017). A Scale for Measuring Consumer Perceptions of Corporate Social Responsibility following the Sustainable Development Paradigm. Journal of Business Ethics, 140(2), 243-262. https://doi.org/10.1111/jonm.12586

Barone, M. J., Miyazaki, A. D. y Taylor, K. A. (2007). The Influence of Cause-Related Marketing on Consumer Choice: Does one Good Turn Deserve Another? Journal of the Academy of Marketing Science, 28(2), 248-262. https://doi.org/10.1177/0092070300282006

Batruch C. (2010). Does CSR make a difference? The Graduate Institute, Geneva. Recuperado de http://www.lundinpetroleum.com/Documents/cr CSR_present_02_10.pdf.

Becker-Olsen, K. L., Cudmore, B. A. y Hill, R. P. (2006). The Impact of Perceived Corporate Social Responsibility on Consumer Behavior. Journal of Business Research, 59(1), 46-53. https://doi.org/10.1016/j.jbusres.2005.01.001

Bridoux, F., Stofberg, N. y Den Hartog, D. (2016). Stakeholders' Responses to CSR Tradeoffs: When Other-Orientation and Trust Trump Material Selfinterest. Frontiers in Psychology, 6, 1992. https://doi.org/10.3389/fpsyg.2015.01992

Brown, T. J. y Dacin, P. A. (1997). The Company and the Product: Corporate Associations and Consumer Product Responses. The Journal of Marketing, 6, 68-84.

Creyer, E H. y Ross, W T. (1997). The Influence of Firm Behavior on Purchase Intention: Do Consumers 
Really Care about Business Ethics? Journal of Consumer Marketing, 14, 421-432.

Dodd, M. D. y Supa, D. W. (2011). Understanding the Effect of Corporate Social Responsibility on Consumer Purchase Intention. Public Relations Journal, 5(3), 1-19.

Du, S., Bhattacharya, C. B. y Sen, S. (2010). Maximizing Business Returns to Corporate Social Responsibility (CSR): The Role of CSR Communication. International Journal of Management Reviews, 12(1), 8-19. https://doi.org/10.1111/j.1468-2370.2009.00276.x

Ferreira, A. I. y Ribeiro, I. (2017). Are you Willing to Pay the Price? The Impact of Corporate Social (IR) Responsibility on Consumer Behavior towards National and Foreign Brands. Journal of Consumer Behaviour, 16(1), https://doi.org/10.1002/cb.1603

Ferreira, D. A., Avila, M. G. y de Faria, M. D. (2010). Corporate Social Responsibility and Customers Perception of Price. Social Responsibility Journal, 6(2), 206-221. https://doi.org/10.1108/17471111011051720

Fisac, R., Moreno, A. M., Mataix, C. y Palacios Fernández, M. (2011). La empresa social: revisión de conceptos y modelo para el análisis organizativo [The Social Enterprise: Review of Concepts and Model for Organizational Analysis]. Revista Española del Tercer Sector, 41-66. Recuperdado de: http://oa.upm.es/12404/

Frederick, W. C. (2016). Commentary: Corporate Social Responsibility: Deep Roots, Flourishing Growth, Promising Future. Frontiers in Psychology, 7, 129. https://doi.org/10.3389/fpsyg.2016.00129

Glavas, A. (2016a). Corporate Social Responsibility and Organizational Psychology: An Integrative Review. Frontiers in Psychology, 7, 144. https://doi.org/10.3389/fpsyg.2016.00144
Glavas, A. (2016b). Corporate Social Responsibility and Employee Engagement: Enabling Employees to Employ more of their whole Selves at Work. Frontiers in Psychology, 7, 796. https://doi.org/ 10.3389/fpsyg.2016.00796

Glavas, A. y Kelley, K. (2014). The Effects of Perceived Corporate Social Responsibility on Employee Attitudes. Business Ethics Quarterly, 24(2), 165202. https://doi.org/10.5840/beq20143206

Glavas, A., Willness, C. R., Jones, D. A. (eds.). (2017). Corporate Social Responsibility and Organizational Psychology: Quid pro Quo. Lausanne: $\quad$ Frontiers Media. https://doi.org/10.3389/978-2-88945-199-9

Gold, A. H., Malhotra, A. y Segars, A. H. (2001). Knowledge Management: An Organizational Capabilities Perspective. Journal of management information systems, 18(1), 185-214. https://doi.org/10.1080/07421222.2001.11045669

Gold, S., Seuring, S. y Beske, P. (2010). Sustainable Supply Chain Management and InterOrganizational Resources: A Literature Review. Corporate Social Responsibility and Environmental Management, 17(4), 230-245. https://doi.org/10.1002/csr.207

Hair, J. F., Black, B. y Babin, B., Anderson, R. E. y Tatham, R. L. (2006). Multivariate data analysis, 6. Londres, Reino Unido: Pearson Educación.

Hameed, I., Riaz, Z., Arain, G. A. y Farooq, O. (2016). How do Internal and External CSR Affect Employees' Organizational Identification? A Perspective from the Group Engagement Model. Frontiers in Psychology, 7, 788. https://doi.org/10.3389/fpsyg.2016.00788

Jones, D. A., Willness, C. R. y Glavas, A. (2017). When Corporate Social Responsibility (CSR) Meets Organizational Psychology: New Frontiers in Micro-CSR Research, and Fulfilling a Quid Pro 
Quo through Multilevel Insights. Frontiers in Psychology, 8, 520. https://doi.org/10.3389/fpsyg.2017.00520

Kotler, P. y Lee, N. (2005). Corporate Social Responsibility: Doing the Most Good for Your Company and Your Cause. Hoboken, NJ: Wiley.

Kusum A. L. y Luan J. (2011). Does Corporate Social Responsibility Build Customer Loyalty? Recuperado de http://adage.com/article/cmostrategy/corporate-social-responsibility-buildcustomer-loyalty/227729/

McWilliams A. y Siegel D. (2001). Corporate Social Responsibility: A Theory of the Firm Perspective. The Academy of Management Review. 26(1),117https://doi.org/127. 10.2307/259398

Meade, A. W. y Craig, S. B. (2012). Identifying Careless Responses in Survey Data. Psychological Methods, 17(3), 437. https://doi.org/10.1037/a0028085

Mohr, L. A. y Webb, D. J. (2005). The Effects of Corporate Social Responsibility and Price on Consumer Responses. Journal of Consumer $A$ ffairs, 39(1), 121-147. https://doi.org/10.1111/j.1745-6606.2005.00006.x

Moreno, A., Uriarte, L. M. y Topa, G. (2010). La responsabilidad social empresarial: Oportunidades estratégicas, organizativas y de recursos humanos [Corporate social responsibility: strategic, organizational and human resources opportunities]. Madrid, España: Pirámide.

Pava, M. L. y Krausz, J. (1996). The Association between Corporate Social-Responsibility and Financial Performance: The Paradox of Social Cost. Journal of Business Ethics, 15, 321-357.

Pérez-López, D., Moreno-Romero, A. y Barkemeyer, R. (2015). Exploring the Relationship between Sustainability Reporting and Sustainability
Management Practices. Business Strategy and the Environment, 24(8), 720-734. https://doi.org/10.1002/bse.1841

Pomering, A. y Dolnicar, S. (2009). Assessing the Prerequisite of Successful CSR Implementation: Are Consumers aware of CSR Initiatives? Journal of Business Ethics, 85, 285-301. https://doi.org/10.1007/s10551-008-9729-9

Quintão, R. T. y Isabella, G. (2015). Corporate Social Responsibility and High and Low-Income Customers: Different Perceptions of Benefit, Value, Price and Purchase Intentions. En L. Robinson (ed.), Marketing Dynamism \& Sustainability, (pp. 801-804). Cham, Switzerland: Springer

Ringle, C. M., Wende, S. y Becker, J. M. (2015). SmartPLS 3. Boenningstedt: SmartPLS GmbH Recuperado de http://www. smartpls. com.

Sen, S. y Bhattacharya, C. B. (2001). Does Doing Good Always Lead to Doing Better? Consumer Reactions to Corporate Social Responsibility. Journal of Marketing Research, 38(2), 225-243. https://doi.org/10.1509/jmkr.38.2.225.18838

Simmons C. J. y Becker-Olsen K. L. (2006). Achieving Marketing Objectives through Social Sponsorships. Journal of Marketing 70(4), 154-169. Recuperado de https://www.jstor.org/stable/30162120

Voliotis, S., Vlachos, P. A. y Epitropaki, O. (2016). Perception-induced Effects of Corporate Social Irresponsibility (CSiR) for Stereotypical and Admired Firms. Frontiers in Psychology, 7, 970. https://doi.org/10.3389/fpsyg.2016.00970

Wang, W., Fu, Y., Qiu, H., Moore, J. H. y Wang, Z. (2017). Corporate Social Responsibility and Employee Outcomes: A Moderated Mediation Model of Organizational Identification and Moral Identity. Frontiers in Psychology, 8, 1906 https://doi.org/10.3389/fpsyg.2017.01906 


\title{
PERCEPTIONS OF CORPORATE SOCIAL RESPONSIBILITY: RELATIONSHIP WITH CUSTOMERS' ATTITUDES AND BEHAVIORS
}

\author{
SERGio A. PÉREZ-MARTÍNEZ ${ }^{1}$ Y GABRIELA TOPA ${ }^{1}$
}

\section{EXTENDED SUMMARY}

\section{Introduction}

Corporate Social Responsibility (hereafter, CSR) is an issue that is gaining more and more interest among companies' managers and, at the same time, awakens academic interest, especially due to its possible impact on the attitudes and behavior of customers. The empirical studies on the relationship between CSR and clients' attitudes and behaviors are varied and most emphasize a close relationship between both variables. For a couple of decades now it has become clear that a negative perception of a company's CSR could have a lasting impact on customers' loyalty and purchase decisions.

Empirical research in Spain on the relationship between the perception of CSR of the company and the attitudes and behavior of customers is scarce and has focused mainly on sectors specifically aimed at providing services. In relation to the IBEX 35 companies, which have high visibility in their respective sectors, important research has been done, but this has been focused on economic perspectives, or the role of external pressures that motivate the practices of RSC.

To sum up, this study proposes that clients' perceptions of corporate CSR, in its three dimensions of Environmental Protection, Social Equity, and Sustainable Economic Development, will be positively related to the perceived value of products or services, with the perceived benefit of products or services and with the intention of buying products or services from the company.

\section{Participants}

The present study has been carried out with the participation of 1011 clients of the IBEX 35 companies. The average age of the participants was 40.4 years (SD: 12.6), $48 \%$ are male, the average age as clients of the company was 14.3 years (DT: 32.3 ).

\section{Procedure}

The participants were recruited from the general population of people over 18 years of age. An invitation email to participate anonymously and voluntarily is sent with the link to the online survey. In accordance with the usual procedures, the pertinent authorization was requested to the Bioethics Committee of the UNED for the present study and this was approved.

\section{Data analyses}

Data were analyzed using Smart PLS v. 3.2.6., a recent software implementation of the PLS approach. The objective of the PLS is predicting dependent variables, latent and manifest, maximizing the explained variance $\left(\mathrm{R}^{2}\right)$ of the dependent variables and minimizing the residual variance of endogen variables in any regression run of the model. To evaluate the model against observed data, an iterative procedure fits observed measures to corresponding latent variables, and then estimates 
relationships among the latent variables. At each stage of the iteration least squares fit, between observed and modeled parameters, is computed and the model is considered as a best-fit solution when the least squares function stabilizes between iterations.

PLS has two strengths that make it well suited to this study. First, PLS was developed to avoid the necessity of large sample sizes and hard assumptions of normality. For this reason, it is often referred to as a form of soft modeling. Although PLS can be used for theory confirmation, it is generally recommended in situations where theory or model is to be built, rather than for confirmation proposes. Second, PLS accounts for measurement error and should provide more accurate estimates of interaction effects such as mediation. Significance was evaluated using bootstrapping of 500 samples of 1011 cases, which leads to a critical t-value of 1.96 for $p<.05$.

\section{Results}

PLS results are presented in two parts: tests of validity and reliability of the measures (outer model), and the tests of hypotheses (inner model). The outer model is the relationships between the manifest indicators and the hypothesized latent constructs.

Reliability of reflective constructs. First, the individual reliability of each indicator is given by loading or correlations between the indicator and the construct $(\lambda)$. Researchers postulate that a latent variable should explain a substantial part of each indicator's variance (usually at least $50 \%$ ). Accordingly, the standardized outer loadings should be higher than .60. Second, the scale reliability allows measuring internal coherency of all indicators in relation with the construct. The composite reliability (hereafter, CR) is a preferred alternative to Cronbach's $\alpha$ as a measure of internal consistency reliability. While Cronbach' s $\alpha$ assumes that all indicators are equally reliable. PLS prioritizes indicators according to their reliability, resulting in a more reliable composite. The acceptable cutoff for CR would be the same as the researcher sets for Cronbach's $\alpha$ since both attempts to measure internal consistency reliability. Consequently,
$\mathrm{CR}$ value should be above .70 , whereas a value below .60 indicates a lack of reliability. Table 2 shows the results of the outer model.

Convergent and discriminant validity. The convergent validity represents the common variance between the indicators and their construct, and it signifies that a set of indicators represents one and the same underlying construct. Authors recommend using the average variance extracted (AVE) as a criterion. The higher AVE value, the more representative are the indicators of the construct on which they load. In general, it is suggested that its value should be above .50. As shown in Table 2, the AVE for each construct was satisfactory. To assess discriminant validity among constructs, the AVE square root should be higher than the squared correlation with all other constructs. Thereby, each construct should share more variance with its own block of indicators than with another construct representing a different block of indicators. Table 1 shows the correlations between the constructs and, along the diagonal, the AVE square root. In view of this data, there is discriminant validity among constructs assessed in the model, despite that intercorrelations among CSR dimensions are high, as could be expected.

\section{Inner model}

The inner model is the structural relationship (i.e. path model) among constructs. It involves an evaluation of the pathways between latent constructs using linear regression in which the regressors can be interpreted as standardized beta coefficients. The confidence intervals of the path coefficients are based on a bootstrapping of 500 samples that permits the generalization of the results and the computation of the Student $t$ for each hypothesis. The essential criterion for assessing the structural model is the coefficient of determination $\left(\mathrm{R}^{2}\right)$ of each endogenous latent variable, which should exceed .10 . The relationships between the Environmental Protection CSR and the intention to purchase are not significant. The same happens with the relations between the CSR of Social Equity and the benefit perceived by the clients. 


\section{Discussion}

The purpose of this study was to empirically analyze the relationship between the perception of CSR by the clients of the IBEX 35 companies with their relationship with the perceived benefit, with the perceived value of the company's services or products, and with the intention of purchase or future acquisition of the product or service by the customers. In view of the results obtained, it can be affirmed that the objective has been reached. The hypothetical model, which has been tested with 1011 clients, shows that the three dimensions - Environmental Protection, Social equity and Sustainable Economic Development - are different from each other. In addition, the results reflect that these perceptions are positively and significantly related to the Perceived Benefit, Perceived Value and purchase intention, although that some discrepant results deserve a more detailed discussion.

First, the findings show that the CSR perceived in its Sustainable Economic Development dimension is the best predictor of the three outcome variables of the model, and that its influence is statistically significant over all of them, although it is higher than the intention of purchase. Second, for the Social Equity dimension, precisely the opposite happens, since it is a predictor of statistical significance lower in its relation to perceived value and purchase intention, while its relationship with perceived benefit lacks significance. The CSR perception of Environmental Protection is a significant predictor of perceived benefit and value, but does not predict significantly the intention to purchase. Given that the two dimensions of CSR related to Environmental Protection and Social Equity have insignificant relationships with the purchase intention, this variable is mostly predicted only by the CSR of Sustainable Economic Development. 
